\title{
The general secretion pathway of Erwinia carotovora subsp. carotovora: analysis of the membrane topology of OutC and OutF
}

\author{
Joanna D. Thomas, ${ }^{2}$ Philip J. Reeves ${ }^{2} \dagger$ and George P. C. Salmond' \\ Author for correspondence: George P. C. Salmond. Tel: +44 1223333 650. Fax : +44 123333345. \\ e-mail: gpcs@mole.bio.cam.ac.uk
}

\footnotetext{
1 Department of Biochemistry, University of Cambridge, Tennis Court Road, Cambridge CB2 1QW, UK

2 Department of Biological Sciences, University of Warwick, Coventry CV4 7AL, UK
}

The out gene cluster of Erwinia carotovora subsp. carotovora (Ecc) encodes the proteins of the type II or general secretory pathway (GSP) apparatus which is required for secretion of pectinase and cellulase. In this study, fusions between Ecc out genes and the topology probe blaM were constructed. The ability of Out protein domains to export BlaM across the cytoplasmic membrane in both Escherichia coli and the cognate host was utilized to confirm the computer-predicted cytoplasmic membrane topology of OutC and OutF. When outC was fused to blaM, the resulting phenotype suggested that the majority of OutC is targeted to the periplasm, typical of a type II bitopic conformation in the cytoplasmic membrane. In contrast, for the outF gene product, three transmembrane regions were identified which connect a large $\mathbf{N}$-terminal cytoplasmic domain, a smaller periplasmic domain, and a large cytoplasmic loop. Fusions between blaM and outD and outE were used to further substantiate the locations of these gene products in the outer membrane and the cytoplasm respectively. The data derived suggest that a number of the Out apparatus components possess domains in the cytoplasm and/or the periplasm with potential for protein-protein interactions which facilitate the secretion of periplasmic enzyme intermediates across the outer membrane to the external milieu.

Keywords : Erwinia carotovora, Out proteins, membrane topology, secretion, $\beta$-lactamase

\section{INTRODUCTION}

The Gram-negative bacterium Erwinia carotovora subsp. carotovora (Ecc) possesses a type II secretion system, alternatively known as the general secretory pathway (GSP), which is highly homologous in a wide range of bacteria (Wharam et al., 1994; Salmond \& Reeves, 1993; Pugsley, 1993). The Erwinia GSP appears to be a two-step mechanism. In the first step, Ecc pectinases (Pel) and cellulases (Cel) are synthesized as precursors with $\mathrm{N}$-terminal signal sequences which are cleaved in a 'Sec-dependent' manner during export across the cytoplasmic membrane to the periplasm. The second step involves secretion across the outer membrane and release of the protein to the extracellular

\footnotetext{
Present address: Department of Biology and Chemistry, Massachusetts Institute of Technology, Cambridge, MA, USA.
}

Abbreviations: Ecc, Erwinia carotovora subsp. carotovora; Echr, Erwinia chrysanthemi; Pel, pectate lyase; Cel, cellulase; BlaM, $\beta$-lactamase. milieu. This second step requires a number of proteinaceous 'membrane traffic wardens' encoded by a genetic cluster conserved in several bacterial genera including Erwinia carotovora, Erwinia chrysanthemi (Echr), Klebsiella oxytoca, Pseudomonas aeruginosa, Xanthomonas campestris and Aeromonas hydrophila (Salmond \& Reeves, 1993; Pugsley, 1993).

The Ecc type II apparatus is encoded by the out cluster, containing 15 out genes termed outB-O and out $S$. The out cluster has been sequenced and Ecc mutants defective for secretion $\left(\mathrm{Out}^{-}\right)$have been isolated (Reeves et al., 1993; S. Wharam, unpublished data). The majority of the Out proteins are predicted to have an inner membrane location based on computer structural predictions of the out gene products. This is a curious phenomenon, since the Out apparatus is believed to form a structure which enables secretion from the periplasm to the exterior of the cell. With the exception of OutF and OutL, the majority of each putative innermembrane Out protein is predicted to protrude into the 
periplasm, an aspect consistent with the notion of formation of an interactive protein structure at the periplasmic face of the cytoplasmic membrane. This inner membrane complex of proteins must interact in some manner with those Out proteins predicted to reside in the outer membrane of the cell (OutD and OutS) to facilitate translocation of the exported Pel and Cel proteins. Therefore, in order to gain an insight into the organization and function of such a putative structure, we are investigating the interactions between a number of the Out proteins and the cytoplasmic membrane. Prior to the elucidation of specific proteinprotein interactions, it is important to determine the precise cellular locations of particular domains of the Out proteins.

Previous work has utilized a series of Ecc out-blaM fusions to assess the ability of Out protein regions to export BlaM across the cytoplasmic membrane in both the cognate host and Escherichia coli, and hence create detailed inner-membrane topology maps of OutG, $-\mathrm{H}$, -I, -J, -K, -L, -N and -O (Reeves et al., 1994; P. Reeves, unpublished data). The resulting phenotype of out $G$, $-H,-I,-I,-K$ and $-N$ blaM fusions suggested that in an E. coli host a type II bitopic conformation in the cytoplasmic membrane is adopted, with the majority of each protein being targeted to the periplasm. OutO is proposed to be a polytopic integral cytoplasmic membrane protein with eight transmembrane domains, connected by three periplasmic and four cytoplasmic loops. OutL appears to have a single transmembrane region with approximately one-third of the protein exposed to the periplasm and two-thirds in the cytoplasm ( $\mathrm{P}$. Reeves, unpublished data). Based on the presence of the type IV pre-pilin consensus sequence in their N-termini, the OutG, $-\mathrm{H},-\mathrm{I}$, and $-\mathrm{J}$ proteins are often termed NMePhe pseudopilins. NMePhe peptidase processing of the NMePhe pseudopilins by Ecc OutO has been demonstrated (Reeves et al., 1994).

Based on amino acid sequence data, Ecc OutC is predicted to adopt a type II bitopic conformation in the cytoplasmic membrane. C-terminal PhoA fusions to the OutC homologues of $K$. oxytoca (PulC) and $P$. aeruginosa $(\mathrm{XcpP})$ have shown that these proteins are located in the cytoplasmic membrane, with the majority of each protein protruding into the periplasm (d'Enfert et al., 1989; Bleves et al., 1996). The presence of multiple hydrophobic domains in $\mathrm{Ecc}$ OutF suggests it to be a polytopic integral cytoplasmic membrane protein. OutE is the only non-hydrophobic protein of the Out apparatus. The presence of two ATP-binding motifs (Walker A and B) in OutE and its homologues implies an ATPase function. Recent work by Sandkvist et al. (1995) suggests that the Vibrio cholerae homologue of OutE, EpsE, interacts with the cytoplasmic domain of the OutL homologue, EpsL. OutE is therefore predicted to be the energizer of the Out apparatus, but its precise function remains elusive. The OutD homologues of the type II secretion systems of $K$. oxytoca (PulD) and $X$. campestris (XpsD) have been shown to form multimers which are localized to the outer membrane of the cell
(Hardie et al., 1996; Hu et al., 1995). The OutD homologue (pIV) from $\mathrm{fl}$-infected $E$. coli exists as a homomultimer of approximately 10 to 12 subunits, and is capable of forming heteromultimers with Echr OutD (Russel \& Kazmierczak, 1993; Kazmierczak et al., 1994). Based on this evidence, it appears that Ecc OutD also forms a multimeric pore structure in the outer membrane of the cell, enabling secretion of periplasmic intermediates of Pel and Cel to the extracellular milieu. The precise locations of OutC, $-\mathrm{D},-\mathrm{E}$, and $-\mathrm{F}$ require further characterization.

In this paper we report further detailed topological analysis of the Ecc type II OutC and OutF proteins utilizing out-blaM fusions. Our results support the prediction that both these proteins are inner-membrane proteins with putative membrane-spanning regions. outD- and outE-blaM fusions have been utilized to substantiate the predicted locations of OutD and OutE in the outer membrane and the cytoplasm respectively.

\section{METHODS}

Bacterial strains and plasmids. The bacterial strains and plasmids used in this study are listed in Table 1 . Relevant characteristics of $p Z C^{\prime}-G^{\prime}$ and $p Z C$ derivatives are described in the text and Fig. 1. Routine DNA manipulation and cloning procedures were performed as described by Sambrook et al. (1989), using the E. coli hosts DH1 and TG1. DNA ligation mixtures were introduced into $E$. coli and supercoiled plasmids were introduced into Ecc SCRI193 using a Bio-R ad Genepulser according to the manufacturer's instructions. All bacterial cultures were grown routinely in $2 \times$ YT broth (Miller, 1972) or nutrient broth agar (NBA) plates (Oxoid). E. coli cells were incubated at $37^{\circ} \mathrm{C}$ and Ecc SCRI193 cells at $30^{\circ} \mathrm{C}$, unless stated otherwise. Where appropriate, media were supplemented with antibiotics at the following concentrations (unless stated otherwise): kanamycin (Kn), $50 \mu \mathrm{g} \mathrm{m}^{-1}$; ampicillin (Ap), $50 \mu \mathrm{g} \mathrm{ml}^{-1}$.

Construction of out-blaM gene fusions. The strategy of Broome-Smith et al. (1990) was used to investigate the topology of OutC and outF. Two recombinant plasmids were constructed, as illustrated in Fig. 1, following methods as described previously by Reeves et al. (1994). To generate blaM fusions to outC, pZC was digested with Xbal and SacI to produce exonuclease III sensitive and resistant sites respectively. Plasmid DNA was treated with a Nested Deletion Kit according to the manufacturer's instructions (Pharmacia) to create progressively truncated forms of outC. DNA samples from deletion time points were digested with EcoRI and ligated with a blaM cassette which was isolated from pYZ5 using PvuII and EcoRI. blaM fusions to OutD, -E and -F were generated in a similar manner by digestion of $\mathrm{pZC}-\mathrm{G}^{\prime}$ with $K p n I$ and HindIII to generate exonuclease III sensitive and resistant sites respectively, and samples from deletion time points were treated with SacI. Ligations were carried out with a blaM cassette isolated from pYZ5 using PvuII and SacI.

Ampicillin resistance of E. coli DH1 and Ecc SCRI193 producing Out-BlaM hybrid proteins. $\mathrm{Kn}^{\mathrm{R}}$ transformants were initially screened for the ability to grow at single-cell density on NBA

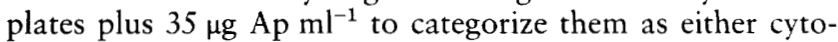
plasmic (negative growth) or periplasmic (positive growth) fusions. Two of the OutE-BlaM fusions were isolated on NBA plates plus $25 \mu \mathrm{g} \mathrm{Ap} \mathrm{ml} \mathrm{g}^{-1}$. Where necessary, fusions were 
Table 1. Bacterial strains and plasmids

\begin{tabular}{|c|c|c|}
\hline Strain/plasmid & Relevant characteristics & Reference/source \\
\hline \multicolumn{3}{|l|}{ Bacterial strains } \\
\hline E. carotovora subsp. carotovora SCRI193 & Wild-type & Laboratory stock \\
\hline E. coli $\mathrm{DH} 1$ & $\begin{array}{l}\text { supE } 44 \text { hsdR } 17 \text { recA1 end } 1 \text { gyrA96 } \\
\text { thi-1 relA1 }\end{array}$ & Hanahan (1983) \\
\hline E. coli TG1 & $\begin{array}{l}\text { supE thi } \Delta(\text { lac-pro }) \text { ecoK hsd } \Delta 5 \\
{\left[\mathrm{~F}^{\prime} \text { traD36 pro } A^{+} B^{+}\right] \text {lacl }{ }^{4} \text { lacZ } \Delta \mathrm{M} 15}\end{array}$ & Gibson (1984) \\
\hline \multicolumn{3}{|l|}{ Plasmids } \\
\hline pYZ4 & $\mathrm{Kn}^{\mathrm{R}}$ lacUV5p & Zhang \& Broome-Smith (1990) \\
\hline pYZ5 & $\mathrm{Tc}^{\mathrm{R}}$ blaM' & Zhang \& Broome-Smith (1990) \\
\hline $\mathrm{pZC}$ & pYZ4 outC & This work (Fig. 1) \\
\hline $\mathrm{pZC}-\mathrm{G}^{\prime}$ & pYZ4 out $C^{\prime}-G^{\prime}$ & This work (Fig. 1) \\
\hline cHIL208 & pHC79 outC-O Ap ${ }^{\mathrm{R}}$ & Reeves et al. (1993) \\
\hline pHCP2 & pBR322: : $\operatorname{lam} B^{+} \mathrm{Ap}^{\mathrm{R}} \mathrm{Tc}^{\mathrm{R}}$ & Clement et al. (1982) \\
\hline
\end{tabular}
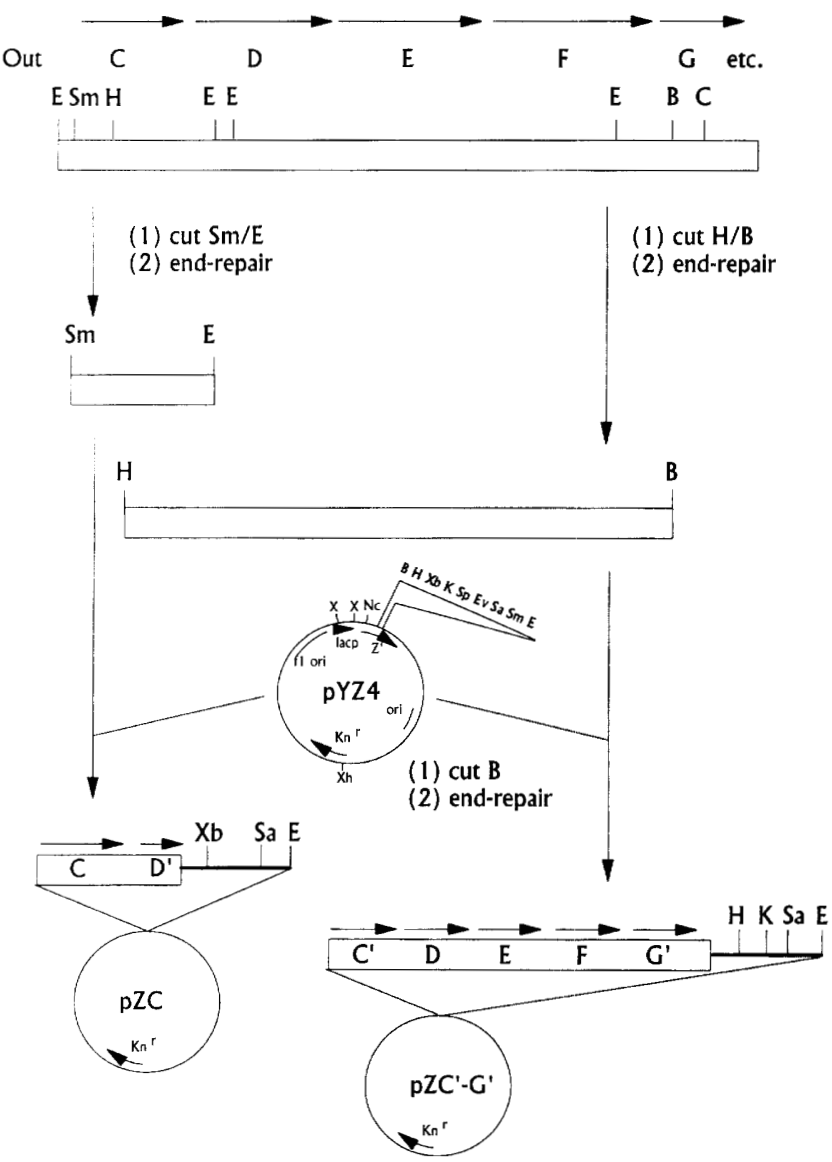

Fig. 1. Construction of $p Z C$ and $p Z C^{\prime}-G^{\prime}$. DNA fragments containing out $C$ and out $C^{\prime}-G^{\prime}$ were obtained from CHIL208 (Reeves et al., 1993). Abbreviations: B, BamHI; C, Clal; E, EcoRl; Ev, EcoRV; H, HindlII; K, Kpnl; Nc, Ncol; Sa, Sacl; lacp, lacUV5 promoter; $Z^{\prime}$, lac $Z \alpha$-peptide coding region.

electroporated into an Ecc SCRI193 host. Subsequently, the minimum inhibitory concentration (MIC) of Ap required to prevent colony formation by single DH1 or SCRI193 cells carrying Out-BlaM fusions was determined using the method described by Edelman $e t$ al. (1987), except that experiments were performed at $30^{\circ} \mathrm{C}$ using NBA.

Nucleotide sequencing of out-blaM fusion junctions. Plasmid DNA was prepared from E. colicells using the Magic Miniprep procedure (Promega) and cycle-sequenced as previously described (Reeves et al., 1994).

Immunoblotting procedures. Total cell protein extracts were prepared, analysed by SDS-PAGE, transferred to nitrocellulose and immunoblotted with anti- $\beta$-lactamase serum (5 prime $\rightarrow 3$ prime, Inc.) as previously described (Reeves et al., 1994).

\section{RESULTS AND DISCUSSION}

\section{Topological analysis of OutC}

The out gene cluster of Ecc SCRI193 has a homologue of the K. oxytoca pulC and Echr outC genes, which both appear to be essential for secretion in their respective host. OutC is predicted to be a type II bitopic cytoplasmic-membrane protein which contains a single hydrophobic region. The hydropathy profile of OutC, investigated by the method of Kyte \& Doolittle (1982) using a window length of 20 residues, indicated a single transmembrane domain (Fig. 2a). The remainder of the protein was highly hydrophilic, suggesting the presence of a large water-soluble domain which probably resides in the periplasm.

To investigate genetically the topology of OutC, fusions between out $\mathrm{C}$ and the topology probe blaM were constructed. The outC gene was cloned into pYZ4 to generate pZC (Fig. 1). Progressive 3' deletions of pZC were generated and fused to blaM from pYZ5. As an initial screen $\mathrm{Kn}^{\mathrm{R}}$ E. coli colonies carrying this fusion series were tested for their ability to grow as patches on NBA plates containing Ap ( $\left.35 \mu \mathrm{g} \mathrm{ml}^{-1}\right)$ when inoculated using a toothpick. The resulting putative outC-blaM in-frame fusions were then tested for their ability to form single colonies $\left(\mathrm{Ap}^{\mathrm{R}}\right)$ on NBA containing Ap $\left(35 \mu \mathrm{g} \mathrm{m}^{-1}\right)$. Plasmid DNA was prepared from $E$. coli colonies carrying putative in-frame fusions and each outC-blaM fusion junction was determined by 

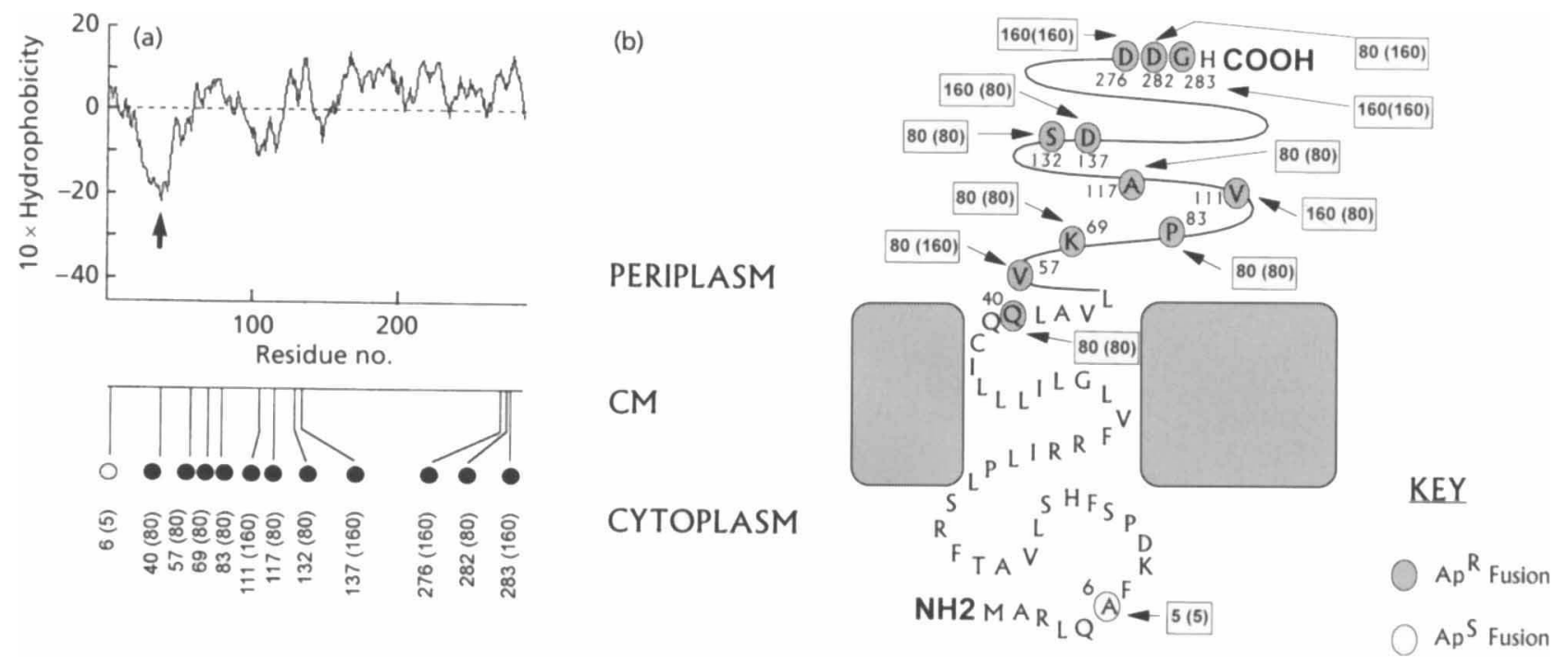

Fig. 2. Hydropathic analysis and proposed two-dimensional membrane topology of OutC. (a) Topological analysis of OutC by the method of Kyte \& Doolittle (1982), using a window of 20 residues. A modified version of the graphics output from the MICROGENIE program (Queen \& Korn, 1984) is displayed. Increasing positive values represent increasing hydrophilicity. The single predicted transmembrane region is indicated by an arrow. The positions of $\mathrm{Ap}^{5}$ (open circle) and $\mathrm{Ap}^{\mathrm{R}}$ (filled circles) BlaM fusion points are shown beneath the hydrophobicity profile and the residue number of each is shown. Numbers in parentheses are MICs ( $\mu \mathrm{g} \mathrm{Ap} \mathrm{ml}^{-1}$ ) using $E$. coli. BlaM fusion positions and MICs (in parentheses) obtained using Ecc are as follows: 6 (5), $40(80), 57(160), 69(80), 83(80), 111(80), 117(80), 132(80), 137(80), 276(160)$, $282(160), 283(160)$. (b) Inner-membrane topology mapping proposes a single transmembrane helix and a C-terminal periplasmic domain. Circles indicate residues which immediately precede a BlaM fusion point as determined by DNA sequencing. Shaded circles correspond to $\mathrm{Ap}^{\mathrm{R}}$ fusions. Numbers adjacent to circles indicate the amino acid residue preceding each BlaM fusion junction. Boxed numbers indicate MIC levels in an E. coli DH1 host (no parentheses) and an Ecc SCRI193 cognate host (parentheses). CM, cytoplasmic membrane.

sequencing analysis using a primer specific to the mature BlaM moiety. Twelve unique OutC-BlaM fusions were isolated in this manner. The derived topology map for OutC is shown in Fig. 2(b).

The level of Ap resistance conferred on single cells is related linearly to the synthesis level of exported forms of $\beta$-lactamase (Jaurin et al., 1982). Therefore, the efficiency of transfer of BlaM by different OutC-BlaM hybrids could be tested by calculating the MIC of Ap required to prevent single colony formation of both an E. coli and a cognate Ecc SCRI193 host carrying each OutC-BlaM fusion protein (Fig. 2b). Eleven fusions conferring an $\mathrm{Ap}^{\mathrm{R}}$ phenotype had $\mathrm{MIC}$ values of

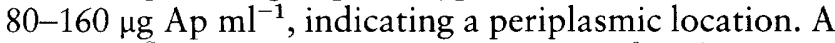
single $\mathrm{Ap}^{\mathrm{s}}$ fusion at amino acid residue $\mathrm{A}^{6}$ had an MIC value of $5 \mu \mathrm{g} \mathrm{Ap} \mathrm{ml} l^{-1}$, suggesting a cytoplasmic location. This residue lies within the predicted signal sequence for sec-dependent export of OutC to the periplasmic face of the cytoplasmic membrane. Presumably, since this fusion does not meet the criteria for a sec-dependent export signal sequence, the first six amino acid residues of OutC are not sufficient to target and anchor BlaM to the periplasmic side of the cytoplasmic membrane. The low MIC value observed for this hybrid protein could merely reflect its stability in comparison to other OutC-BlaM hybrid proteins. In order to investigate this, total extracts from Ecc SCRI193 cells expressing each OutC-BlaM fusion were analysed by SDS-PAGE and immunoblotting using an anti- $\beta$ - lactamase antibody. All OutC-BlaM fusions were stable in an Ecc background, including the $\mathrm{Ap}^{\mathrm{s}} \mathrm{A}^{6}$ fusion (Fig. $3 a)$, suggesting that the MIC values observed are a true indication of the extent of BlaM transfer to the periplasm.

The OutC homologues of the K. oxytoca (PulC) and $P$. aeruginosa $(\mathrm{XcpP})$ type II secretion systems have previously been characterized using PhoA fusion technology. d'Enfert et al. (1989) described two unique inframe PulC-PhoA fusions which encoded active alkaline phosphatase. One of these fusions primarily fractionated with the inner membrane upon isopycnic sucrose density-gradient centrifugation of membrane vesicles. Recent work by Bleves et al. (1996) generated a PhoA fusion directly downstream of the $\mathrm{L}^{173}$ residue of the $P$. aeruginosa OutC homologue, $\mathrm{XcpP}$. This fusion also encoded active alkaline phosphatase, suggesting that the C-terminus of the protein protrudes into the periplasm in a similar manner to that of PulC. The authors also showed that overexpression of the $x c p P$ gene resulted in the localization of $\mathrm{XcpP}$ in the inner-membrane fraction upon subcellular fractionation. Both of these studies utilized the non-cognate host, E. coli. The detailed topological analysis described here has now confirmed that Ecc OutC also adopts a type II bitopic conformation in the cytoplasmic membrane in both the non-cognate host, E. coli, and the cognate host, Ecc. The topology mapping data strongly correlate with the predictions from the hydropathy profile of OutC. The majority of 


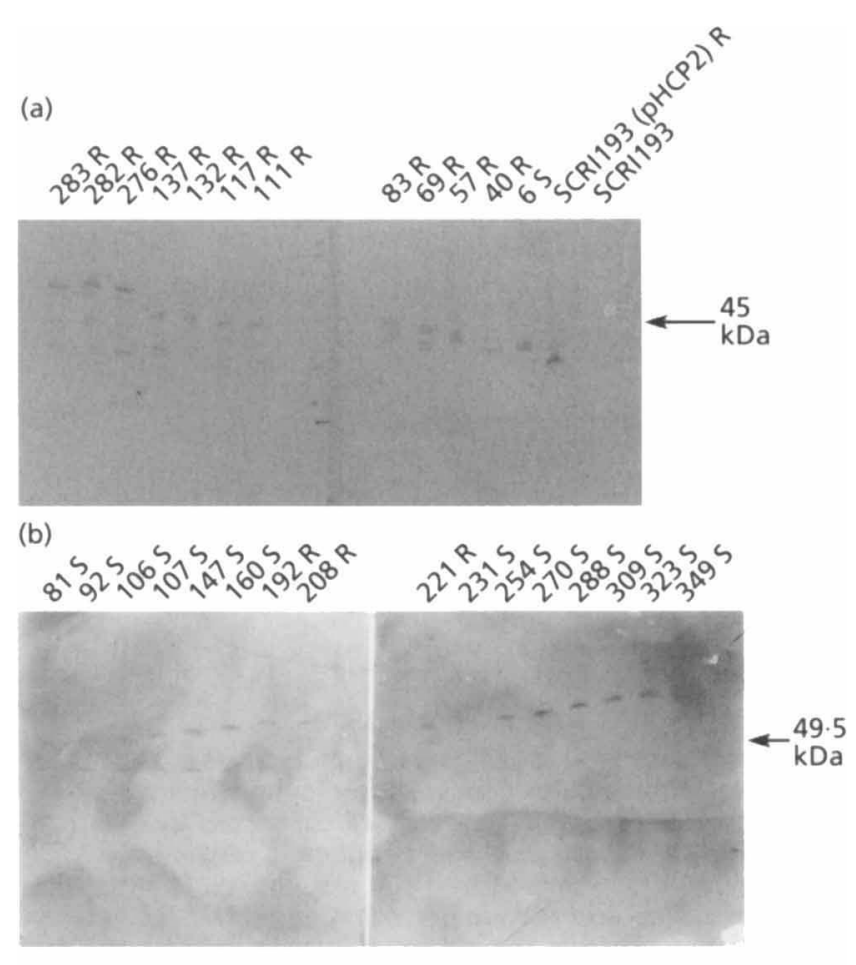

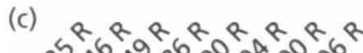

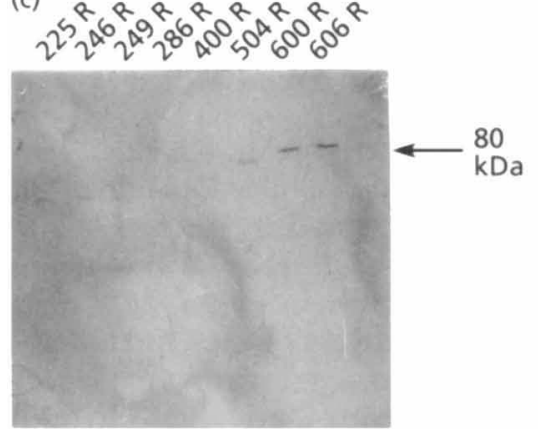

Fig. 3. Visualization of Out-BlaM hybrid proteins. Whole-cell protein samples were separated on a $12.5 \%$ SDS-PAGE Phast gel (Pharmacia) and Out-BlaM fusion proteins were detected as described in Methods. The location of the Out-BlaM fusion point in hybrid proteins and the phenotype they produced in an Ecc SCRI193 host are shown above the corresponding lane; $R, A p^{R} ; S, A p^{5}$. (a) OutC-BlaM fusions. The location of a $45 \mathrm{kDa}$ marker is indicated by an arrow. (b) OutF-BlaM fusions. The location of a $49.5 \mathrm{kDa}$ marker is indicated by an arrow. (c) OutD-BlaM fusions. The location of an $80 \mathrm{kDa}$ marker is indicated by an arrow.

OutC appears to exist as a hydrophilic domain which protrudes into the periplasm. The location of OutC suggests that this protein may play an interactive role in the formation of a membrane-spanning complex at the periplasmic face of the cytoplasmic membrane. Therefore, OutC has potential for protein-protein interactions with the majority of the other cytoplasmicmembrane-located or outer-membrane-located Out proteins and/or the periplasmic intermediate forms of the secreted extracellular enzymes (Pel or Cel) themselves.

\section{Topological analysis of OutF}

OutF is predicted to be a polytopic integral cytoplasmicmembrane protein. The hydropathy profile of OutF was investigated by the method of Kyte \& Doolittle (1982) using a window length of 20 residues. The profile indicated three transmembrane regions and three highly hydrophilic domains (Fig. 4a). To confirm the locations of the latter, we aimed to determine the membrane topology of OutF.

In order to investigate the membrane topology of out $F$ a fragment of DNA containing outD, $-E$ and $-F$ was cloned into pYZ4 to produce pZC'-G' (Fig. 1). Progressive $3^{\prime}$ deletions of $p Z C^{\prime}-\mathrm{G}^{\prime}$ were generated and fused to blaM. The resulting fusion series was characterized in a similar manner to that of OutC, resulting in the isolation of 53 unique OutF-BlaM fusions with either an $\mathrm{Ap}^{\mathrm{S}}$ or an $\mathrm{Ap}^{\mathrm{R}}$ phenotype. For simplicity the relative position of each fusion with respect to the hydropathic profile (Fig. 4a) is not shown, but a detailed topology map for OutF is shown in Fig. 4(b).

The efficiency of transfer of BlaM by 24 OutF-BlaM hybrids was tested by calculating the MIC of Ap required to prevent single colony formation of an E. coli host carrying each OutF-BlaM fusion protein. Ten of the most appropriate fusions, based on the hydrophobicity data, were also characterized in the wild-type host, Ecc SCRI193 (Fig. 4b). MIC values were generally either $80 \mu \mathrm{g} \mathrm{Ap} \mathrm{ml} l^{-1}$ (indicative of a periplasmic location) or $5 \mu \mathrm{g} \mathrm{Ap} \mathrm{ml} l^{-1}$ (indicative of a cytoplasmic location) in an $E$. coli host. In the wild-type host, MIC values were routinely lower than in the E. coli host, but followed the same overall pattern. This was analogous to all other Out proteins characterized by topological analysis to date. This phenomenon is probably due to interactions between the Out-BlaM hybrid and the wild-type Ecc Out apparatus. $\mathrm{L}^{231}$ of OutF is predicted by the OutF hydrophobicity profile to reside in the second transmembrane domain, yet it was found to have an MIC value of $20 \mu \mathrm{g} \mathrm{Ap} \mathrm{ml} l^{-1}$ in an E. coli host and $5 \mu \mathrm{g} \mathrm{Ap} \mathrm{ml}^{-1}$ in Ecc. Presumably the truncated nature of this domain in the $\mathrm{L}^{231}$-BlaM hybrid protein is insufficient to transfer the BlaM moiety back into the cytoplasm, resulting in its partial protrusion into the periplasm, at least in the non-cognate host, E. coli. Nevertheless, topological analysis has clearly confirmed the presence of three transmembrane domains in OutF which connect a single periplasmic domain and two cytoplasmic domains.

Total extracts from Ecc SCRI193 cells producing a selection of the OutF-BlaM hybrids were analysed by immunoblotting in a similar manner as for OutC-BlaM fusions. All OutF-BlaM hybrids analysed, with the exception of $\mathrm{L}^{231}-\mathrm{BlaM}$, were stable in an Ecc background, suggesting that the corresponding MIC values are a true indication of the extent of BlaM transfer (Fig. 3b). The quantity of the $\mathrm{L}^{231}$-BlaM hybrid (Fig. 3b, lane 10) appeared to be lower than that of the other OutF-BlaM hybrids. Therefore it is possible that $\mathrm{L}^{231}-\mathrm{BlaM}$ is an unstable periplasmic hybrid. This 


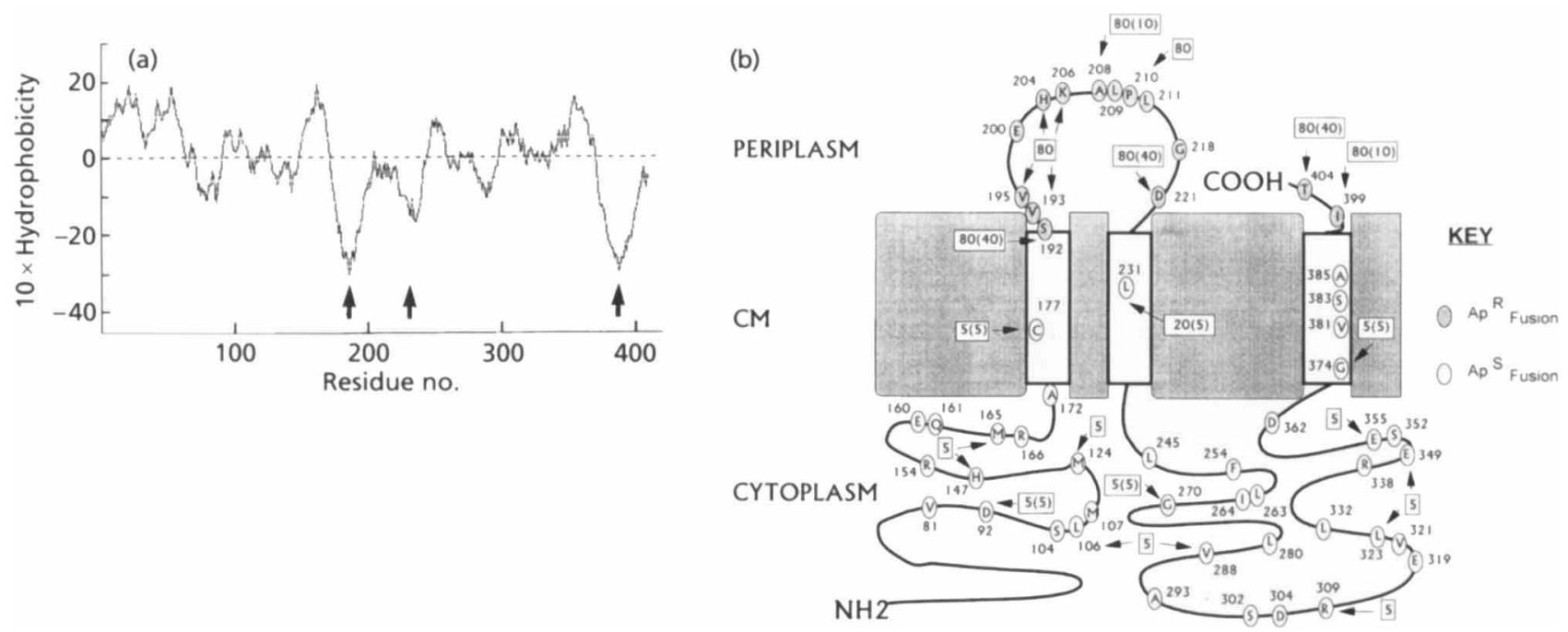

Fig. 4. Hydropathic analysis and proposed two-dimensional membrane topology of OutF. (a) Topological analysis of OutF was performed and is displayed as described in Fig. 2. The locations of three putative transmembrane regions are indicated by arrows. (b) Inner-membrane topology mapping proposes three transmembrane helices which are connected by one periplasmic and two cytoplasmic loops. The $\mathrm{N}$ - and $\mathrm{C}$-termini appear to be cytoplasmic and periplasmic respectively. Circles indicate residues which immediately precede a BlaM fusion point as determined by DNA sequencing. Shaded circles correspond to $\mathrm{Ap}^{R}$ fusions. Numbers adjacent to circles indicate the amino acid residue preceding each BlaM fusion junction. Boxed numbers indicate MIC levels in an E. coli DH1 host (no parentheses) and an Ecc SCRI193 cognate host (parentheses). CM, cytoplasmic membrane.

would explain the increased sensitivity to Ap observed in both E. coli and Ecc hosts in comparison to other periplasmic-located outF-BlaM fusions.

OutF-BlaM topological analysis confirmed the twodimensional prediction of the hydropathy profile. OutF is a polytopic inner-membrane protein with a small perisplasmic loop and two larger cytoplasmic domains connected by three transmembrane regions. Therefore, unlike OutC, OutF is a potential candidate for interactions on both faces of the cytoplasmic membrane.

\section{Out-BlaM fusion analysis of OutD and OutE}

Two members of the out cluster, outD and outE, encode proteins which are predicted to reside in the outer membrane and the cytoplasm respectively. The hydropathy profiles of OutD and OutE were investigated by the method of Kyte \& Doolittle (1982) using a window length of 20 residues. OutD appeared to be highly hydrophobic throughout, further characterizing it as a membrane protein (Fig. 5a). In contrast, OutE is highly hydrophilic, indicative of its predicted cytoplasmic location (Fig. 5b). blaM fusions to outD and outE arose by default from the topological analysis of OutF using the construct $\mathrm{pZC}-\mathrm{G}^{\prime}$. Therefore, these fusions were characterized by both MIC analysis and immunoblot detection to further elucidate the putative locations of OutD and OutE. It must be noted that only cytoplasmic membrane proteins can be truly topologymapped in this manner. Although OutD and OutE are not predicted to be inner-membrane proteins, the MIC profiles of OutD- and OutE-BlaM hybrids were assumed to be indicative of their cellular locations. The MIC values of ten OutD-BlaM and three OutE-BlaM hybrids in an E. coli host are shown below the corresponding hydropathy profile in Fig. 5. MIC data for the OutE-BlaM hybrids and six of the OutD-BlaM hybrids in an Ecc SCRI193 host are shown in the legend of Fig. 5. The high MIC values for OutD-BlaM hybrids suggest a location in either the periplasm or the outer membrane. This, combined with the highly hydrophobic profile of OutD, authenticates its outer membrane location. In contrast, the low MIC values of OutE-BlaM fusions are characteristic of a cytoplasmic position.

Out-BlaM hybrids were shown to be stable in an Ecc background via immunoblot detection with anti- $\beta$ lactamase antisera (Fig. 3c). A similar detection showed that the OutE-BlaM fusions were quite unstable (data not shown). Two of the OutE fusions could only be isolated by decreasing the concentration of $\mathrm{Ap}$ in detection plates to $25 \mu \mathrm{g} \mathrm{ml}^{-1}$. In addition, only three OutE fusions were isolated in total. These observations suggest that OutE-BlaM fusions can be toxic to the cell when free in the cytoplasm. In summary, we have provided further evidence of the outer-membrane and cytoplasmic locations of OutD and OutE respectively.

\section{Concluding remarks}

In this report we provide detailed topological analyses of OutC, $-\mathrm{D},-\mathrm{E}$ and $-\mathrm{F}$, four proteins encoded by the Ecc SCRI193 out cluster. All four proteins appear to be 

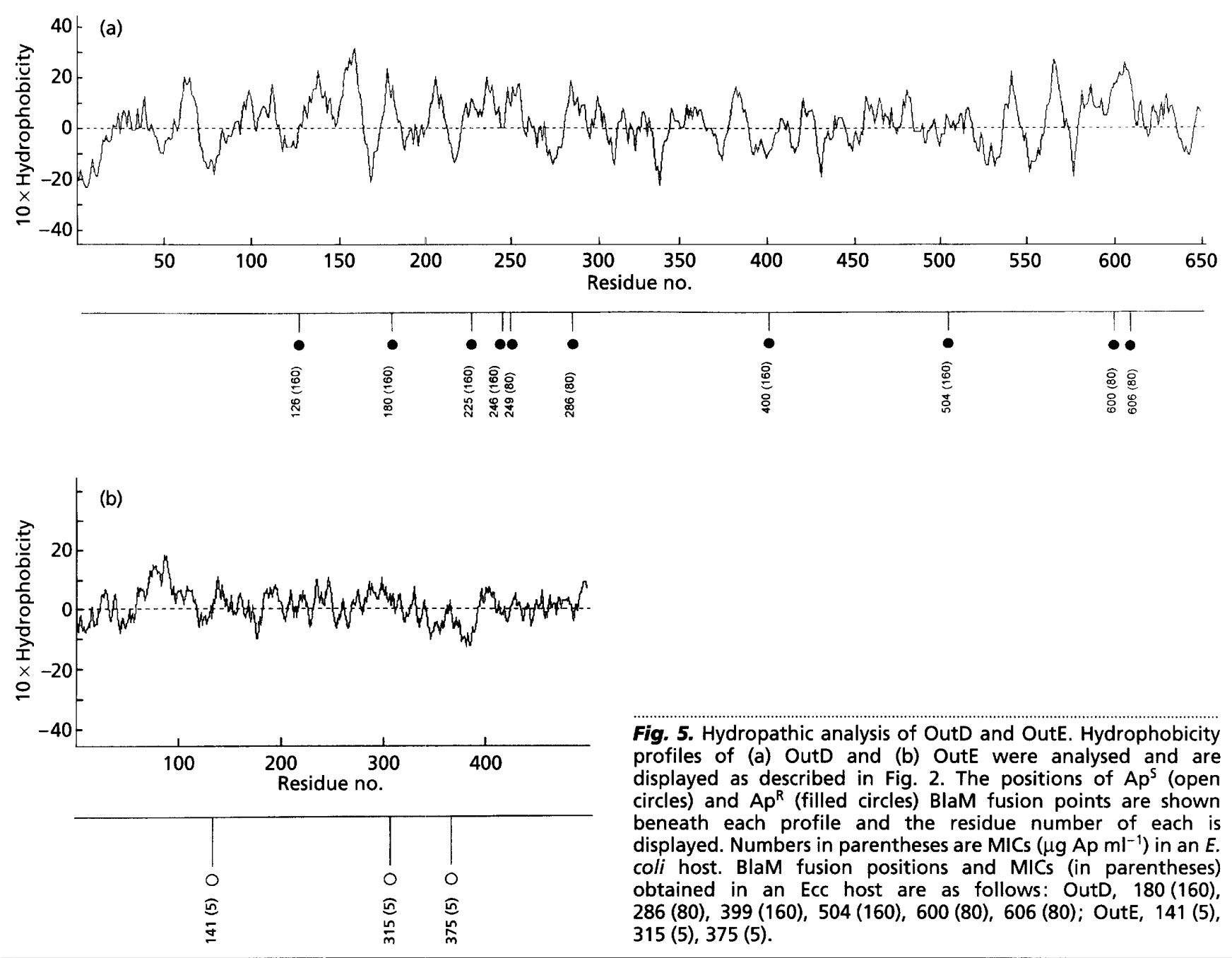

Fig. 5. Hydropathic analysis of OutD and OutE. Hydrophobicity profiles of (a) OutD and (b) OutE were analysed and are displayed as described in Fig. 2. The positions of $\mathrm{Ap}^{\mathrm{s}}$ (open circles) and $A p^{R}$ (filled circles) BlaM fusion points are shown beneath each profile and the residue number of each is displayed. Numbers in parentheses are MICs $\left(\mu \mathrm{g} \mathrm{Ap} \mathrm{ml}^{-1}\right)$ in an $E$. coli host. BlaM fusion positions and MICs (in parentheses) obtained in an Ecc host are as follows: OutD, $180(160)$, $286(80), 399(160), 504(160), 600(80), 606(80)$; OutE, $141(5)$, $315(5), 375(5)$.

highly homologous in a number of other bacterial systems, and essential for secretion of Pel and Cel in Ecc SCRI193. The data we present support structural predictions, based on hydropathy, that OutC is a cytoplasmic membrane protein with a single transmembrane domain and a large hydrophilic periplasmic domain. Our data strongly suggest that OutF is also a cytoplasmic membrane protein, but with three transmembrane domains, a small periplasmic loop, a large cytoplasmic loop and an N-terminal cytoplasmic domain. The putative outer membrane and cytoplasmic locations of OutD and OutE have been further verified.

Topological two-dimensional structure analysis of a number of Out proteins has facilitated the prediction of domains which may be functionally interacting with other Out proteins, or the secreted enzymes themselves. Recent evidence has shown that OutC and OutD are the only non-interchangeable components of the Ecc and Echr Out systems, suggesting that OutC and OutD are candidate gatekeepers of species-specific secretion of proteins via the type II pathway (Lindeberg et al., 1996). The extreme $\mathrm{N}$-terminal domain of OutD is less widely conserved in its homologous counterparts than the C- terminus of the protein. It is suspected that this $\mathrm{N}$ terminal domain protrudes into the periplasm, where it potentially interacts with other members of the type II secretion apparatus. Is Erwinia Out species-specificity dependent on a direct protein-protein interaction between the N-terminal domain of OutD and the periplasmic domain of OutC?

Recently, Sandkvist et al. (1995) have provided evidence that in the homologous type II apparatus of Vibrio cholerae EpsL interacts with EpsE in the cytoplasm. The authors also tentatively suggest that EpsF may have a minor role in EpsE interaction. The presence of domains in OutF and OutL which are located to both the cytoplasm and the periplasm clearly indicates the potential involvement of these proteins in interactions on both faces of the cytoplasmic membrane. In this laboratory, a series of point mutations in the out cluster have been generated by localized hydroxylamine mutagenesis (J. Housby, unpublished results). The Out ${ }^{\text {ts }}$ phenotype of a mutation in the cytoplasmic domain of OutL suggests that conformational changes in this region prevent secretion of extracellular enzymes. Furthermore, in trans complementation has confirmed the 
isolation of two OutF mutants with an Out ${ }^{-}$phenotype (J. Thomas, unpublished results).

From this study it is predicted that OutC and OutF interact with other members of the Out apparatus and/or the secreted enzymes themselves, to enable secretion across the outer membrane. Therefore, we speculate that OutF, in conjunction with OutL, plays some interactive role with OutE in order to mediate energy transduction from this ATPase across the cytoplasmic membrane. In turn, this interaction may cause a conformational change in the periplasmic domains of the Out proteins anchored to the cytoplasmic membrane, for example OutC or OutL, enabling the opening of a multimeric OutD pore structure in the outer membrane. Thus the secretion of periplasmic enzyme intermediates across the periplasmic space and the outer membrane could be enabled.

Future work will involve further characterization of a number of $\mathrm{Out}^{-}$point mutations and subsequent suppressor isolation. In this manner, we hope to investigate the possible protein-protein interactions which allow secretion of periplasmic Pel and Cel intermediates to the external milieu.

\section{ACKNOWLEDGEMENTS}

We wish to thank Jenny Broome-Smith for $\mathrm{pYZ} 4 / 5$ and for technical advice on using this system. Susie Wharam and Vincent Mulholland are acknowledged for their helpful discussion. This work was supported by award number 88/P03042 from BBSRC. J. D. Thomas was funded by a BBSRC studentship.

\section{REFERENCES}

Bleves, S., Lazdunski, A. \& Filloux, A. (1996). Membrane topology of three $\mathrm{Xcp}$ proteins involved in exoprotein transport by Pseudomonas aeruginosa. J Bacteriol 178, 4297-4300.

Broome-Smith, J. K., Tadayyon, M. \& Zhang, Y. (1990). $\beta$ Lactamase as a probe of membrane protein assembly and protein export. Mol Microbiol 4, 1637-1644.

Clement, J. M., Perrin, D. \& Hedgpeth, J. (1982). Analysis of a receptor and $\beta$-lactamase synthesis and export using cloned genes in a minicell system. Mol Gen Genet 185, 302-310.

d'Enfert, C., Reyss, I., Wandersman, C. \& Pugsley, A. P. (1989). Protein secretion by Gram-negative bacteria. Characterization of two membrane proteins required for pullulanase secretion by Escherichia coli K-12. J Biol Chem 264, 17462-17468.

Edelman, A., Bowler, L., Broome-Smith, J. K. \& Spratt, B. G. (1987). Use of a $\beta$-lactamase fusion vector to investigate the organization of penicillin-binding protein $1 \mathrm{~B}$ in the cytoplasmic membrane of Escherichia coli. Mol Microbiol 1, 101-106.

Gibson, T. J. (1984). Studies on Epstein-Barr virus genome. $\mathrm{PhD}$ thesis, Cambridge University, England.

Hanahan, D. (1983). Studies on transformation of Escherichia coli with plasmids. J Mol Biol 166, 557-580.

Hardie, K. R., Lory, S. \& Pugsley, A. P. (1996). Insertion of an outer membrane protein in Escherichia coli requires a chaperone-like protein. $E M B O J 15,978-988$.

Hu, N., Hung, M., Liao, C. \& Lin, M. (1995). Subcellular location of
$\mathrm{XpsD}$, a protein required for extracellular protein secretion by Xanthomonas campestris pv. campestris. Microbiology 141 , 1395-1406.

Jaurin, B., Grundström, T. \& Normark, S. (1982). Sequence elements determining $a m p C$ promoter strength in Escherichia coli. EMBO J 1, 875-881.

Kazmierczak, B. I., Mielke, D. L., Russel, M. \& Model, P. (1994). pIV, a filamentous phage protein that mediates phage export across the bacterial cell envelope, forms a multimer. $J \mathrm{Mol}$ Biol 238, 187-198.

Kyte, J. \& Doolittle, R. F. (1982). A simple method for displaying the hydrophobic character of a protein. J Mol Biol 157, 105-132.

Lindeberg, M., Salmond, G. P. C. \& Collmer, A. (1996). Complementation of deletion mutations in a cloned functional cluster of Erwinia chrysanthemi out genes with Erwinia carotovora out homologues reveals OutC and OutD as candidate gatekeepers of species-specific secretion of proteins via the type II pathway. Mol Microbiol 20, 175-190.

Miller, J. H. (1972). Experiments in Molecular Genetics. Cold Spring Harbor, NY: Cold Spring Harbor Laboratory.

Pugsley, A. (1993). The complete general secretory pathway in Gram-negative bacteria. Microbiol Rev 57, 50-108.

Queen, C. \& Korn, L. J. (1984). A comprehensive sequence analysis programme for the IBM personal computer. Nucleic Acids Res $12,581-599$.

Reeves, P. J., Whitcombe, D., Wharam, S., Gibson, M., Allison, G., Bunce, N., Barallon, R., Douglas, P., Mulholland, V., Stevens, S., Walker, D. \& Salmond, G. P. C. (1993). Molecular cloning and characterization of 13 out genes from Erwinia carotovora subspecies carotovora: genes encoding members of a general secretion pathway (GSP) widespread in Gram-negative bacteria. Mol Microbiol 8, 433-456.

Reeves, P. J., Douglas, P. \& Salmond, G. P. C. (1994). The general secretion pathway of Erwinia carotovora subspecies carotovora: analysis of the cytoplasmic membrane topology of seven Out proteins using a $\beta$-lactamase topology probe. Mol Microbiol 12, 445-457.

Russel, M. \& Kazmierczak, B. (1993). Analysis of the structure and subcellular location of filamentous phage pIV. J Bacteriol 175, 3998-4007.

Salmond, G. P. C. \& Reeves, P. J. (1993). Membrane traffic wardens and protein secretion in Gram-negative bacteria. Trends Biochem Sci 18, 7-12.

Sambrook, J., Fritsch, E. F. \& Maniatis, T. (1989). Molecular Cloning: a Laboratory Manual, 2nd edn. Cold Spring Harbor, NY: Cold Spring Harbor Laboratory.

Sandkvist, M., Bagdasarian, M., Howard, S. P. \& Dirita, V. J. (1995). Interaction between the autokinase EpsE and EpsL in the cytoplasmic membrane is required for extracellular secretion in Vibrio cholerae. EMBO J 14, 1664-1673.

Wharam, S. D., Mulholland, V. \& Salmond, G. P. C. (1994). Conserved virulence factor regulation and secretion systems in bacterial pathogens of plants and animals. Eur J Plant Pathology 101, 1-13.

Zhang, Y. \& Broome-Smith, J. K. (1990). Correct insertion of a simple eukaryotic plasma-membrane protein into the cytoplasmic membrane of Escherichia coli. Gene 96, 51-57.

Received 9 August 1996; revised 7 October 1996; accepted 15 October 1996. 